\title{
Valoración de la eficacia de la malla de polipropileno en la reparación del prolapso urogenital en 106 pacientes
}

\author{
Zapardiel Gutiérrez I, De la Fuente Valero J, Iniesta Pérez S, Botija Botija J, Pérez Medina T, \\ Bajo Arenas JM.
}

Departamento de Obstetricia y Ginecología. Hospital Universitario Santa Cristina. Madrid.

Actas Urol Esp. 2008;32(8):821-826

\section{RESUMEN}

VALORACIÓN DE LA EFICACIA DE LA MALLA DE POLIPROPILENO EN LA REPARACIÓN DEL PROLAPSO UROGENITAL EN 106 PACIENTES

Objetivo: evaluar la eficacia y seguridad de las mallas de polipropileno en la corrección del prolapso urogenital. Material y método: estudio retrospectivo, no randomizado sobre 106 pacientes que desde Abril de 2005 a Enero de 2007 se sometieron a corrección de diferentes tipos de prolapso urogenital mediante malla de polipropileno. El seguimiento se realizó mediante visitas en consulta a los 2 y 6 meses. Las variables a analizadas fueron: edad, paridad, presencia de menopausia, tipo de intervención, tiempos quirúrgicos y de hospitalización y complicaciones. Posteriormente se realizó un análisis descriptivo de los datos.

Resultados: La edad media fue de 64,4 años. De las 106 pacientes, 97 eran multíparas (91,51\%) y 98 habían pasado la menopausia $(92,45 \%)$. La intervención mayoritaria fue la colocación de una malla anterior con banda libre de tensión para la corrección de la incontinencia urinaria asociada representando un 34,90\% del total. Se obtuvo una tasa global de complicaciones intraoperatorias del 2,83\%, inmediatas del 37,73\% y tardías del 21,69\%. La tasa de éxito del procedimiento a los 6 meses estuvo entre 80-100\% según el tipo de intervención.

Conclusiones: Existe una baja tasa de complicaciones intraoperatorias y a los 6 meses en las cirugías de reparación del suelo pélvico en los prolapsos urogenitales con la malla de polipropileno, considerando este método como una opción eficaz y muy segura para el tratamiento esta patología.

Palabras clave: Prolapso. Cistocele. Incontinencia.

\section{ABSTRACT \\ EFECTIVENESS EVALUATION OF POLYPROPYLENE MESH IN THE REPAIR OF UROGENITAL PROLAPSE IN 106 PATIENTS}

Objetive: to evaluate the efficacy and security of polypropylene meshes in the repair of urogenital prolapse.

Material and method: retrospective and non-randomized study in 106 patients which had different kinds of urogenital prolapse repaired using polypropylene meshes between April 2005 and January 2007. The follow-up was carried out by two visits to the hospital, 2 and 6 months after surgery. The variables analyzed were age, parity, menopause presence, kind of surgical technique, surgical time, time at hospital and complications. Afterwards, the information was analyzed descriptively.

Results: average age was 64.4 years. The rate of multiparity and menopause women was $91.51 \%$ and $92.45 \%$ respectively. The most used surgical technique was the anterior mesh with tension-free band (34.90\%), to repair the associated urinary incontinence. The rate of intraoperatory complications was $2.83 \%$, immediate complications was $37.73 \%$ and late complications was $21.69 \%$. The success rate after 6 months was $80-100 \%$ depending on the technique.

Conclusions: there is a low rate of intraoperatory and 6 months after the repair in the reconstructive surgery of pelvic floor for the urogenital prolapses using polypropylene meshes, which makes this technique a secure and effective option for the treatment of this problem.

Keywords: Prolapse. Cystocele. Incontinence. 
$\mathrm{S}_{\mathrm{p}}^{\mathrm{e}}$ estima que el riesgo que tiene una mujer de padecer a lo largo de su vida una intervención de prolapso urogenital es del $11,1 \%{ }^{1,2}$. El prolapso urogenital es una entidad que se estima que afecta entre $1-43 \%{ }^{2}$ de las pacientes tras una histerectomía, la dificultad en la estimación de este dato reside en que a las pacientes afectas les cuesta acudir al profesional por ser un problema embarazoso para ellas, además el porcentaje de recurrencia tras la cirugía varía desde el $24 \%$ al $43 \%$ según la técnica quirúrgica empleada ${ }^{3}$.

Se han descrito multitud de técnicas en la literatura científica para la reparación de los prolapsos del suelo pélvico desde culdoplastias como la de McCall $^{4}$ y uteropexias a través de los ligamentos uterosacros como la descrita por Shull et al. ${ }^{5}$ hasta intervenciones más agresivas como sacrocolpopexias abdominales o algo menos como intervenciones laparoscópicas. Desde hace varios años tenemos a nuestra disposición una nueva arma terapéutica para la reparación del suelo pélvico que parece ofrecer grandes ventajas frente a sus predecesores: las mallas sintéticas ${ }^{4}$.

El objetivo de nuestro estudio es evaluar la eficacia y seguridad de las mallas de polipropileno en la corrección del prolapso urogenital.

\section{MATERIAL Y MÉTODOS}

Realizamos un estudio retrospectivo, no randomizado sobre 106 pacientes que desde Abril de 2005 a Enero de 2007 se sometieron a corrección quirúrgica de diversos tipos de prolapso urogenital, con un seguimiento máximo de 6 meses.

A cada paciente se la evaluó de manera conjunta por un ginecólogo y seguidamente por un urólogo recogiendo en una base de datos la información sobre la anamnesis, exploración física y pruebas complementarias como la analítica completa de sangre y orina, el estudio urodinámico y la ecografía transvaginal. Con todo esto se estableció la indicación del tipo de malla según el grado (según la estandarización internacional de POP-Q) y características del prolapso urogenital para cada paciente concreta.

Además se recopiló toda la información acerca del tipo de cirugía, tiempos quirúrgicos, tiempo de hospitalización y complicaciones tanto intraoperatorias e inmediatas como tardías, considerando intraoperatorias las surgidas durante el acto quirúrgico, inmediatas desde la salida de quirófano hasta la fecha de alta hospitalaria y tardías las surgidas después del alta.

También se les realizó a los 2 y 6 meses de la cirugía una revisión de la corrección urogenital mediante una exploración en consulta, en esta se catalogó de corrección exitosa del prolapso cuando se observó la restitución anatómica del mismo sin recidivas del prolapso, así mismo se evaluaron complicaciones tardías como pudieron ser las extrusiones de malla.

Se colocaron 3 tipos de mallas correctoras de polipropileno Ginecare Prolift ${ }^{\circledR}$ (Johnson \& Johnson): anterior (Fig. 1) (PA), posterior (PP) y total (PT). Asociadas o no a histerectomía vaginal (HTV) y/o corrección de la incontinencia urinaria con Ginecare TVT Obturador System ${ }^{\circledR}$ (TOT).

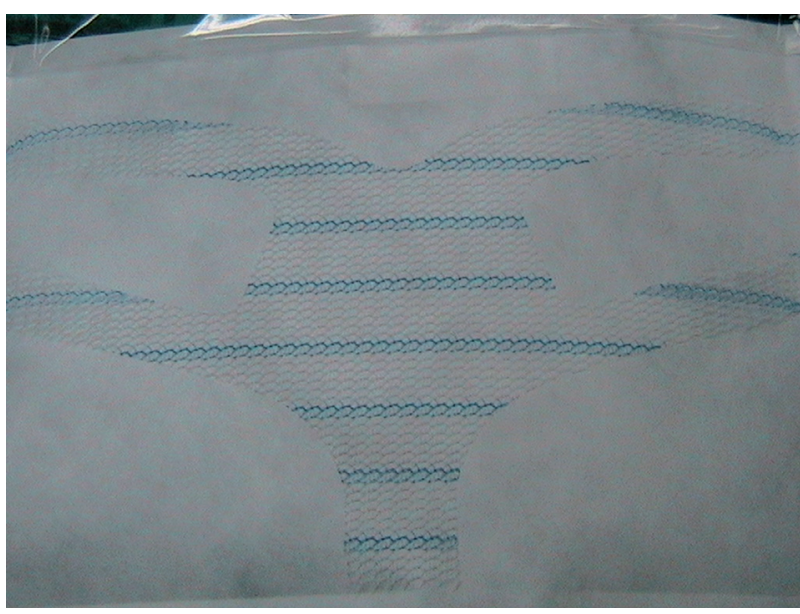

FIGURA 1. Malla de polipropileno Prolift ${ }^{\circledR}$ anterior.

La colocación de las mallas reparadoras del suelo pélvico así como las TOT se realizaron según la técnica habitual, usando los aplicadores del kit para su disposición vía vaginal (Fig. 2) y siguiendo las instrucciones del fabricante (Fig. 3). Todas las histerectomías se realizaron por vía vaginal, mediante la técnica de Péham-Amreicht. Las interveciones fueron llevadas a cabo por un cirujano experimentado.

El análisis de los datos se realizó usando el paquete estadístico SPSS 13.0. Usando para la estadística descriptiva la media, desviación estándar y rango. Comprobando la distribución normal de los datos de la muestra con el test de KolmogorovSmirnov. 


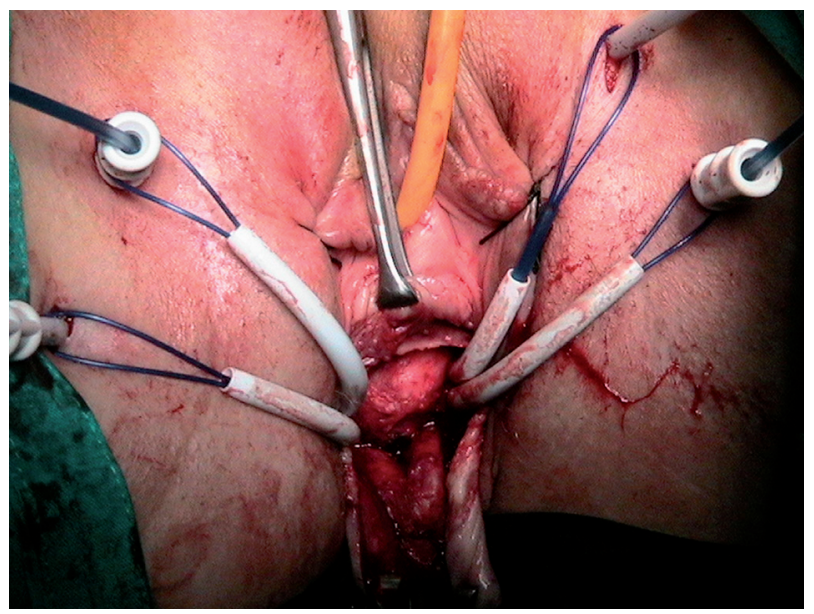

FIGURA 2. Colocación malla Prolift $\circledast$ anterior con aplicadores del kit.

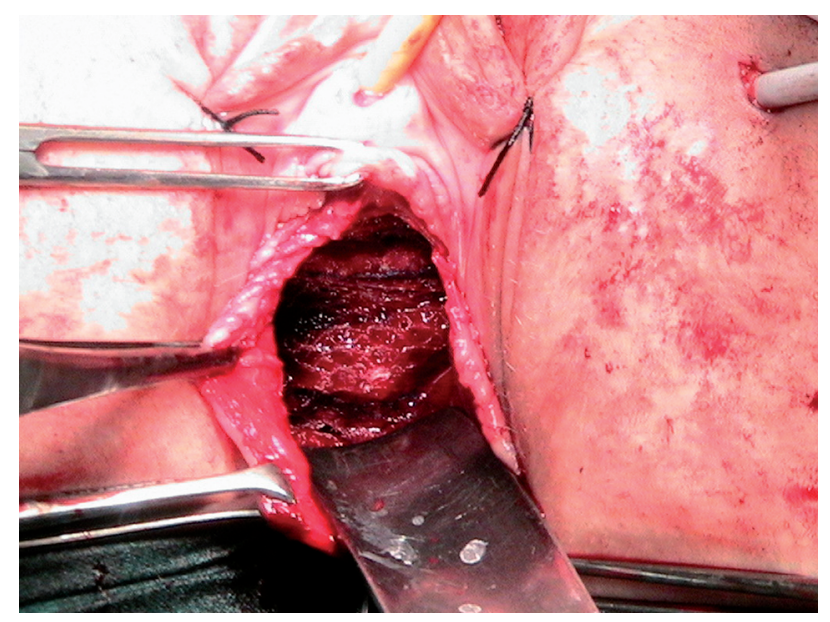

FIGURA 3: Malla de polipropileno Prolift ${ }^{\circ}$ anterior colocada.

\section{RESULTADOS}

Nuestra población de estudio consta de 106 pacientes con una edad media \pm desviación estándar de 64,4 \pm 9,90 años (rango 37 - 81 años). De las 106 pacientes, 97 eran multíparas (91,51\%) y 98 habían pasado la menopausia $(92,45 \%)$.

Se realizaron 57 PA (53,77\%), 36 PT (33,96\%) y 13 PP $(12,27 \%)$, distribuídos en relación a la realización de HTV y/o TOT como muestra la Tabla 1. Cabe destacar que la intervención mayoritaria fue la colocación de una malla PA + TOT representando un $34,90 \%$ del total.

Los tiempos quirúrgicos se muestran en la Tabla 2 así como los tiempos de hospitalización, destacando una media de $132,60 \pm 21.89$ minutos en la realización de un PT + HTV + TOT y la estancia media de 4,7 \pm 1,34 días del PA + HTV + TOT que fueron los de mayor duración de media.
Tabla 1. Distribución y tipos de técnicas realizadas.

\begin{tabular}{llcc}
\hline & & $\begin{array}{c}\text { Número } \\
\text { absoluto }\end{array}$ & $\begin{array}{c}\text { Porcentaje } \\
\text { del total }\end{array}$ \\
\hline PA & solo & 5 & 4,71 \\
& + HTV & 4 & 3,77 \\
& + TOT & 37 & 34,90 \\
& +HTV+TOT & 11 & 10,37 \\
PT & solo & 4 & 3,77 \\
& +HTV & 2 & 1,88 \\
& +TOT & 14 & 13,20 \\
& +HTV+TOT & 16 & 15,15 \\
PP & solo & 2 & 1,88 \\
& +HTV & 0 & 0 \\
& +TOT & 11 & 10,37 \\
\multirow{2}{*}{ TOTAL } & +HTV+TOT & 0 & 0 \\
\hline
\end{tabular}

PA: prolift anterior; PT: prolift total; PP: prolift posterior; HTV: histetrectomia vaginal; TOT: banda transobturadora para incontinencia urinaria

En la Tabla 3 vienen reflejados el número de complicaciones intraoperatorias, inmediatas y tardías por tipo de operación ya sean leves o graves, obteniendo una tasa global de complicaciones intraoperatorias del 2,83\%, inmediatas del 37,73\% y tardías del 21,69\%.

De las 3 complicaciones intraoperatorias registradas, 2 correspondieron a sangrado (que se resolvieron mediante sutura de los vasos sangrantes) y la tercera corresponde al olvido de una gasa que se subsanó antes del alta de la paciente del hospital. Las 40 complicaciones inmediatas (a veces distintas complicaciones en la misma paciente) fueron debidas a las siguientes causas: 5 infecciones del tracto urinario, 9 fueron dolor en la zona de la cirugía, hubo 9 hematomas y 4 sangrados, 2 pacientes tuvieron edema de la zona, 1 astenia, 4 incontinencia de orina (estás pacientes se reevaluaron y entraron en el protocolo normal de incontinencia urinaria, ya que 3 fueron de nueva a parición y no llevaban asociada TOT y 1 fue por fallo de la técnica), 1 disquecia, 2 retención urinaria (que se resolvió mediante sondajes temporales, acabando cediendo la retención en el plazo máximo de dos semanas) y uno síndrome vasovagal. Se presentó de forma temprana una extrusión de malla, que consiste en la erosión de la mucosa vaginal y exposición de la malla al exterior, que se reparó quirúrgicamente y una erosión vaginal por el material protésico subyacente, sin llegar a extrusionarse, que también se reparó mediante cirugía. La técnica de reparación de las extrusiones consiste en recortar el fragmento 
Tabla 2. Tiempos quirúrgicos y de hiospitalización según tipo de intervención

\begin{tabular}{|c|c|c|c|c|c|c|c|}
\hline & & \multicolumn{3}{|c|}{$\begin{array}{c}\text { Tiempos quirúrgico } \\
\text { (minutos) }\end{array}$} & \multicolumn{3}{|c|}{$\begin{array}{c}\text { Tiempo Hospitalización } \\
\text { (dias) }\end{array}$} \\
\hline & & Media & $\begin{array}{l}\text { Desviación } \\
\text { estándar }\end{array}$ & Rango & Media & $\begin{array}{c}\text { Desviación } \\
\text { estándar }\end{array}$ & Rango \\
\hline \multirow[t]{4}{*}{ PA } & solo & 58,40 & 4,22 & 55-65 & 2,5 & 0,71 & $2-3$ \\
\hline & $+\mathrm{HTV}$ & 116,25 & 22,87 & $90-145$ & 3 & 1 & $2-4$ \\
\hline & +TOT & 67,33 & 14,80 & 47-95 & 3 & 1,37 & $2-8$ \\
\hline & +HTV+TOT & 99,73 & 21,92 & $65-145$ & 4,7 & 1,34 & $3-7$ \\
\hline \multirow[t]{3}{*}{ PT } & $\begin{array}{l}\text { solo } \\
+\mathrm{HTV}\end{array}$ & 110,00 & 4,08 & $105-115$ & 3 & 0 & $3-3$ \\
\hline & +TOT & 117,85 & 27,82 & 85-165 & 3,87 & 1,64 & $2-7$ \\
\hline & +HTV+TOT & 132,60 & 21,89 & $95-175$ & 4,28 & 1,38 & $2-8$ \\
\hline \multirow[t]{2}{*}{ PP } & $\begin{array}{l}\text { solo } \\
+\mathrm{HTV}\end{array}$ & & & & & & \\
\hline & $\begin{array}{l}+ \text { TOT } \\
+ \text { +HTV+TOT }\end{array}$ & 90,00 & 26,27 & $65-140$ & 2,33 & 0,52 & $2-3$ \\
\hline
\end{tabular}

PA: prolift anterior; PT: prolift total; PP: prolift posterior; HTV: histetrectomia vaginal; TOT: banda transobturadora para incontinencia urinaria

Tabla 3. Complicaciones de la técnica segun tipo de intervención

\begin{tabular}{|c|c|c|c|c|}
\hline & & $\begin{array}{l}\text { Complicaciones } \\
\text { intraoperatorias }\end{array}$ & $\begin{array}{l}\text { Complicaciones } \\
\text { inmediatas }\end{array}$ & $\begin{array}{c}\text { Complicaciones } \\
\text { tardias }\end{array}$ \\
\hline \multirow[t]{4}{*}{ PA } & solo & $0 / 5$ & $2 / 5$ & $1 / 5$ \\
\hline & $+\mathrm{HTV}$ & $0 / 4$ & $0 / 4$ & $0 / 4$ \\
\hline & +TOT & $1 / 37$ & $11 / 37$ & $6 / 37$ \\
\hline & +HTV+TOT & $0 / 11$ & $5 / 11$ & $0 / 11$ \\
\hline \multirow[t]{4}{*}{ PT } & solo & $0 / 4$ & $2 / 4$ & $1 / 4$ \\
\hline & $+\mathrm{HTV}$ & $0 / 2$ & $1 / 2$ & $0 / 2$ \\
\hline & $+\mathrm{TOT}$ & $1 / 14$ & $8 / 14$ & $5 / 14$ \\
\hline & +HTV+TOT & $0 / 16$ & $7 / 16$ & $4 / 16$ \\
\hline \multirow[t]{2}{*}{ PP } & $\begin{array}{l}\text { solo } \\
+\mathrm{HTV}\end{array}$ & $0 / 2$ & $2 / 2$ & $1 / 2$ \\
\hline & $\begin{array}{l}+ \text { TOT } \\
+ \text { HTV+TOT }\end{array}$ & $1 / 11$ & $2 / 11$ & $5 / 11$ \\
\hline \multicolumn{2}{|c|}{ TOTAL } & $2,83 \%$ & $37,73 \%$ & $21,69 \%$ \\
\hline
\end{tabular}

PA: prolift anterior; PT: prolift total; PP: prolift posterior; HTV: histetrectomia vaginal; TOT: banda transobturadora para incontinencia urinaria.

de malla que está empezando a erosionar la mucosa vaginal o bien ya se ha extruido, refrescando los bordes quirúrgicos y cerrando la mucosa vaginal con sutura reabsorbible.

De las complicaciones tardías, que fueron 23 , nos encontramos con dos incontinencias de orina (de nueva aparición entrando en el protocolo de evaluación de incontinencia urinaria habitual de nuestro centro), 8 extrusiones que se repararon en un segundo tiempo quirúrgico sin incidencias, 8 prolapsos, 1 absceso de cúpula y otro perirrectal, 2 hematomas y 1 paciente con dolor crónico y dispareunia.

Por último en la Tabla 4 se puede observar la alta tasa éxito de cada cirugía a los 2 y 6 meses de su realización. intervención incontinencia urinaria.

\section{DISCUSION}

Si repasamos la literatura científica, podemos encontrar numerosos artículos en los que las complicaciones en la colocación de mallas sintéticas vaginales son minimas ${ }^{6-8}$, predominando de entre todas las complicaciones tres que tienen una incidencia mayor: el fracaso en la corrección del prolapso que puede llegar a alcanzar valores desde $6-8 \%^{9,10}$ hasta el $43 \%^{3}$, extrusión del material protésico y erosiones de la malla con cifras de alrededor del $7 \%$ y dispareunia que puede llegar al 12\%9,10.

Tabla 4. Tasa de éxito a los 2 y 6 meses según tipo de

\begin{tabular}{llcc}
\hline & & $\begin{array}{c}\text { Tasa éxito a los } \\
\text { 2 meses (\%) }\end{array}$ & $\begin{array}{c}\text { Tasa éxito a los } \\
\mathbf{6} \text { meses(\%) }\end{array}$ \\
\hline PA & solo & 100,00 & 100,00 \\
& +HTV & 100,00 & 100,00 \\
& +TOT & 84,84 & 86,21 \\
& +HTV+TOT & 88,88 & 85,71 \\
$\mathrm{PT}$ & solo & 100,00 & 100,00 \\
& +HTV & 100,00 & 100,00 \\
& +TOT & 75,00 & 88,88 \\
& +HTV+TOT & 100,00 & 100,00 \\
PP & solo & 50,00 & 50,00 \\
& +HTV & & \\
& +TOT & 60,00 & 62,50 \\
& +HTV+TOT & & \\
\hline
\end{tabular}

PA: prolift anterior; PT: prolift total; PP: prolift posterior; HTV: histetrectomia vaginal; TOT: banda transobturadora para 
Si comparamos las tasas de complicaciones más frecuentes descritas en el párrafo anterior con las obtenidas en nuestro estudio, se puede observar que la cantidad de complicaciones observadas no es despreciable, aunque sí cabe reseñar que la gran mayoría de ellas fueron leves y transitorias, pudiendo considerar aceptables un $2,83 \%$ de precoces, $37,73 \%$ de inmediatas (de las cuales quizá las 2 retenciones urinarias merezcan la pena recalcar, siendo la tasa real de complicaciones importantes menor del 5\%) y $21,69 \%$ de complicaciones tardías (que parece el grupo más importante ya que incluyen 8 prolapsos y 8 extrusiones de malla). Si nos centramos en las tres complicaciones no leves más frecuentes, observamos que la tasa de recurrencia del prolapso en nuestras pacientes fue de 7,54\%, encontrándose en el rango más bajo (6-8\%) de este tipo de complicación encontrado en la literatura científica; la de extrusiones de la malla con reparación quirúrgica de las mismas fue del 9,43\% que sí es ligeramente más alta que la de otras series encontradas (que se acercan al 7\%) aunque es un aumento que no consideramos significativo; y de dispareunia y dolor a los 6 meses de $0,94 \%$ que si es sustancialmente más baja que en la mayoría de series publicadas. Resulta llamativo como el número de complicaciones aumenta cuando la colocación de la malla va acompañada de histerectomía vaginal o de TOT, tanto en las mallas anteriores, como posteriores y totales. Quizás este aumento de complicaciones tenga más relación con el aumento de tiempos quirúrgicos, que como se puede observar en la Tabla 2 suele ser mayor ala añadir mas procedimientos.

Por lo tanto podemos observar que la tasa de complicaciones de nuestro estudio es muy parecida a las de otros trabajos nos centramos en las complicaciones sustanciales, siendo más baja la de dispareunia. El motivo de que nuestras tasas global de complicaciones resulte tan elevada es que hemos incluido todo tipo de complicaciones, incluso banales, actitud que no está reflejada en los trabajos con los que nos hemos comparado.

Las tasas satisfactorias de complicaciones importantes que hemos observado puede deberse a varios factores: en primer lugar el sistema
Ginecare Prolift $^{\circledR}$ permite una mayor tensión sobre los tejidos herniados gracias al sistema de sustentación a través de brazos ajustables en el momento de su colocación; el segundo lugar, comparado con otras técnicas, esta es menos invasiva, permitiendo mantener la integridad de otras estructuras adyacentes. Por último la malla usada es macroporo (poros mayores de 75 micrones) y monofilamento, la primera característica permite mayor flexibilidad y adaptabilidad facilitando además el paso de células inflamatorias y factores de crecimiento lo que mejora si biointegración y disminuye la tasa de infecciones. El monofilamento también favorece la disminución del riesgo de infección al no poderse quedar acantonados los agentes infecciosos entre las hebras de los materiales trenzados. A disminuir este riesgo se puede ayudar con antibioterapia profiláctica prequirúrgica y con un taponamiento vaginal impregnado con antiséptico posquirúrgico.

Nuestros resultados son acordes a lo esperado y reflejado en la literatura internacional e indican que existe una tasa de complicaciones aceptable a los 2 y 6 meses en las cirugías de reparación del suelo pélvico en los prolapsos urogenitales con la malla de polipropileno Ginecare Prolift ${ }^{\circledR}$, considerando este método como una opción segura y que merece la pena teniendo en cuenta la alta tasa de éxito que se consigue con este procedimiento.

La tasa de éxito entre las distintas cirugías a los dos meses estuvo en torno al 80-100\% (excepto en el PP que fue alrededor del 60\%) y a los 6 meses fue la misma lo que indica una alta eficacia de la técnica (algo menos en el PP) que unida a su seguridad hacen de ella una opción de posible elección para este tipo de trastornos del suelo pélvico. Parece que ala asociar otros procedimientos como la histerectomía vaginal o la TOT la eficacia de la malla baja algo, también pudiendo estar relacionado con la intervención más cruenta y el aumento de tiempos quirúrgicos.

La corrección del prolapso urogenital con malla de polipropileno es un método eficaz y seguro. Tiene una tasa de complicaciones aceptable y alta tasa de éxito a los 6 meses aunque hacen falta estudios a largo plazo para confirmar los buenos resultados obtenidos con esta técnica. 


\section{REFERENCIAS}

1. Olsen AL, Smith VJ, Bergstrom JO, Colling JC, Clark AL. Epidemiology of surgically managed pelvic organ prolapse and urinary incontinence. Obstet Gynecol 1997;89(4):501-506.

2. Weber AM, Richter HE. Pelvic organ prolapse. Obstet Gynecol. 2005;106(3):615-634.

3. Käser O, Ikle FA, Hirsch HA. Atlas of gynecological surgery, Thieme-Stratton Inc. New York (1985).

4. Birch C. The use of prosthetics in pelvic reconstructive surgery. Best Pract Res Clin Obstet Gynaecol. 2005;19(6):979-991.

5. Shull BL, Bachofen C, Coates KW, Kuehl TJ. A transvaginal approach to repair of apical and other associated sites of pelvic organ prolapse with uterosacral ligaments. Am J Obstet Gynecol. 2000;183(6):1365-1373.

6. Mourtzinos A, Raz S. Repair of vaginal vault prolapse and pelvic floor relaxation using polypropylene mesh. Curr Opin Obstet Gynecol. 2006;18(5):555-559.

7. Gauruder-Burmester A, Koutouzidou P, Rohne J, Gronewold M, Tunn R. Follow-up after polypropylene mesh repair of anterior and posterior compartments in patients with recurrent prolapse. Int Urogynecol J Pelvic Floor Dysfunct. 2007;18(9):10591064.
8. Amrute KV, Eisenberg ER, Rastinehad AR, Kushner L, Badlani GH. Analysis of outcomes of single polypropylene mesh in total pelvic floor reconstruction. Neurol Urodyn. 2007;26(1):53-58.

9. Baessler K, Maher CF. Mesh augmentation during pelvic-floor reconstructive surgery: risks and benefits. Curr Opin Obstet Gynecol. 2006;18(5):560-566.

10. De Tayrac R, Devoldere G, Renaudie J, Villard P, Guilbaud O, Eglin G. Prolapse repair by vaginal route using a new protected low-weight polypropylene mesh: 1-year functional and anatomical outcome in a prospective multicentre study. Int Urogynecol J Pelvic Floor Dysfunct. 2007;18(3):251-256.

Correspondencia autor: Dr. I. Zapardiel Gutiérrez Departamento de Obstetricia y Ginecología

Hospital Universitario Santa Cristina

O`Donell 59 - 28009 Madrid

Tel.: 915574300

E-mail autor: ignaciozapardiel@hotmail.com

Información artículo: Original - Incontinencia urinaria femenina

Trabajo recibido: febrero 2008

Trabajo aceptado: marzo 2008 